https://doi.org/10.21670/ref.2117080

Artículos

\title{
La producción social de la frontera Chiapas- Guatemala: estado del arte
}

\section{Social production of the Chiapas-Guatemala border: the current state of affairs}

\author{
Enriqueta Lerma** (D) https://orcid.org/0000-0003-3422-3547 \\ Perla Mónica Castro Cruz ${ }^{b}$ (D) https://orcid.org/0000-0003-3082-2227
}

a Universidad Nacional Autónoma de México, Centro de Investigaciones Multidisciplinarias sobre Chiapas y la Frontera Sur, México, correo electrónico: elermaro@unam.mx

${ }^{\mathrm{b}}$ Universidad Nacional Autónoma de México, Facultad de Ciencias Políticas y Sociales, México, correo electrónico: pmonicastro@gmail.com

\section{Resumen}

El objetivo del artículo es ofrecer una revisión sistematizada de los estudios realizados sobre la producción social de la frontera sur. En especial se enfatiza en las indagaciones sobre el tramo Chiapas-Guatemala, publicadas desde la década de 1990. La bibliografía que se presenta tiene en común el incluir aspectos que permiten comprender la dinámica social en los territorios fronterizos, más allá de la mirada

Recibido el 16 de febrero de 2021. Aceptado el 22 de octubre de 2021. Publicado el 1 de noviembre de 2021.

* Autora para correspondencia: Enriqueta Lerma. Correo electrónico: elermaro@unam.mx

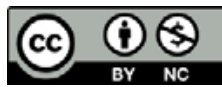

Esta obra está protegida bajo una Licencia Creative Commons Atribución-NoComercial 4.0 Internacional. de los flujos migratorios. Se concluye la necesidad de profundizar en estudios que permitan comprender la vida de los habitantes permanentes de la frontera sur.

Palabras clave: frontera sur, frontera Chiapas-Guatemala, ordenamiento territorial, seguridad nacional, Guardia Nacional.

\section{Abstract}

The objective of the article is to offer a systematized review of the studies carried out on the social production of the southern border. In particular, it is emphasized in the inquiries on the Chiapas-Guatemala section, published since the 1990s. The bibliography presented has in common the inclusion of aspects that allow us to understand the social dynamics in the border territories, beyond the perspective of migratory flows. The need to deepen studies that allow understanding the life of the permanent inhabitants of the southern border is concluded.

Keywords: southern border, Chiapas-Guatemala border, territorial organization, national security, National Guard.

CÓMO CITAR: Lerma, E. \& Castro Cruz, P. M. (2021). La producción social de la frontera Chiapas-Guatemala: estado del arte [Social production of the Chiapas-Guatemala border: the current state of affairs]. Estudios Fronterizos, 22, e080. https://doi.org/10.21670/ ref. 2117080 


\section{Introducción}

En un estudio publicado en 2007, resultado de una metodología participativa para la elaboración de mapas mentales sobre México, Alfredo Guerrero (2007) observó que los participantes (estudiantes mexicanos) dibujaban la frontera norte con Estados Unidos como un espacio cerrado, definido y cargado de significados adversos, mientras que la delimitación territorial con Guatemala y Belice aparecía nebulosa, rural y abierta. No había claridad respecto a su longitud o forma, ni sobre su origen en la historia. Menos se mencionaba algo relacionado con la Comisión Internacional de Límites y Aguas entre México y Guatemala. No podía ser de otro modo: en la mentalidad mexicana el muro fronterizo estaba al norte y no al sur. Esta noción de muro al norte y amplitud sureña, sin embargo, ya no se mantiene. Se ha transformado de 2000 a 2020. La idea de una frontera al sur "que debe controlarse" está más presente. Las noticias sobre nuevos dispositivos que buscan frenar la migración centroamericana son constantes y atraen la mirada de la opinión pública, especialmente la de un gran número de investigadores y estudiantes. De modo que, en la actualidad, si repitiéramos el ejercicio de Alfredo Guerrero, el trazo de la frontera sur sería otro y ya no se vería tan amable.

Algunos acontecimientos han sido relevantes en el reforzamiento de las fronteras a escala global. Entre ellos, el más significativo fue el ataque a las Torres Gemelas en Nueva York el 11 de septiembre de 2001, que implicó la instrumentación de nuevos procesos securitivos internacionales. Como se sabe, tras esta coyuntura, México se vio presionado a terminar el siglo xx e iniciar el xxı intensificando la seguridad territorial: por un lado, para menguar su propia emigración a Estados Unidos y, por otro, para controlar los flujos centroamericanos en tránsito por su territorio. Sin embargo, fue hasta la segunda década del siglo xxi que el objetivo se puso en evidencia en el tramo fronterizo Chiapas-Guatemala. El develo fue posible gracias a dos procesos muy difundidos: uno, el detectar a numerosos niños migrantes que viajaban solos del Triángulo del Norte Centroamericano a Estados Unidos, ${ }^{1}$ registrados de 2010 a 2016, y denunciados por organismos de derechos humanos. El segundo, de data reciente, la emergente e inesperada organización de caravanas migrantes, que ingresaron a México por el Soconusco, a partir de 2018. Ambas coyunturas empujaron el interés por la frontera sur y atrajeron a decenas de investigadores y estudiantes a esta región, para fortalecer así las indagaciones, especialmente bajo el eje de migración.

Es de señalar que, aunque migración y frontera son temas relacionados, en el caso de la frontera Chiapas-Guatemala, las investigaciones se han centrado en el primer tema y menos en el modo en que se ha ido produciendo la frontera como un territorio con características propias. Así que la instrumentación de dispositivos de control fronterizo en territorio limítrofe ha sido poco abordada. Por parte del grupo de investigación La producción de la frontera Chiapas-Guatemala: una aproximación al ordenamiento territorial y a su resignificación, de corte socioantropológico, ha privilegiado indagar sobre el segundo punto, interrogando sobre el modo en que se ha producido el ordenamiento territorial en la frontera sur desde 2000 hasta 2021, a partir de la política securitiva y recuperando la opinión que al respecto mantienen los habitantes de esta región, más allá de la mirada de los migrantes en tránsito.

\footnotetext{
${ }^{1}$ El Triángulo del Norte Centroamericano es el nombre con que se conoce en conjunto a tres países:
} Guatemala, Honduras y EI Salvador. 
Como parte de la indagación, se ha revisado bibliografía especializada, planes y programas del gobierno federal, convenios internacionales y algunos informes de organizaciones no gubernamentales y asociaciones civiles, en la búsqueda por adentrarse en el contexto social reciente de la frontera, e interpretar la percepción de la población local sobre el ordenamiento territorial y su impacto en la vida cotidiana. Han sido vastas las políticas propuestas, pero también muchos los planes abortados por diversas causas. Conscientes de ello, el interés es rastrear cuáles planes y programas han tenido repercusión en la producción de una frontera controlada. Principalmente aquellos que han provocado transformaciones detectadas por sus habitantes en la cotidianidad.

Con relación a este interés y con el deseo de contribuir en la comprensión del proceso, se ha redactado el presente estado del arte, derivado del proyecto, con el objetivo de ofrecer pistas que faciliten el camino a sociólogos y antropólogos interesados en iniciar una investigación sobre la producción de la actual frontera sur en el tramo Chiapas-Guatemala. El texto se organiza en dos partes: $a$ ) en la primera, se hace una revisión de los estudios realizados sobre temas fronterizos a partir de la década de $1990, b$ ) en la segunda parte se reseñan resultados de pesquisas sobre el ordenamiento territorial y la lógica de la seguridad nacional. Se mencionan los principales resultados publicados por especialistas sobre la región y se recurre también a ciertos convenios, planes y programas que deben revisarse para profundizar en el análisis de la instrumentación de la frontera sur.

\section{Estudios especializados}

En el estudio de la conformación de la frontera sur se cuenta con una larga serie de estudios históricos que abarcan la época prehispánica, transitan a la época colonial y se debaten en los intersticios de la delimitación fronteriza en la época posindependentista. No es intención de este artículo remitir a la complejidad de este largo proceso, sino que se restringe a resultados puntuales, que ofrecen pistas para comprender eventos recientes y que dieron pie a la actual instrumentación fronteriza.

Para comprender la conformación de la frontera sur de México a nivel regional es recomendable revisar el libro coordinado por Phillipe Bovin (1997), Las fronteras del Istmo. Fronteras y sociedades entre el sur de México y América Central, obra que permite, a través de la pluma de diversos especialistas, situar la delimitación del territorio mexicano en el contexto centroamericano y caribeño. En esta obra, fundamentalmente geopolítica, se pueden encontrar datos históricos que permiten conocer el proceso de formación de las fronteras nacionales en la región, las aspiraciones políticas locales de integración territorial y la influencia de las fuerzas políticas internacionales en la configuración actual. Asimismo, aborda distintas aristas demográficas, migratorias, comerciales, culturales y extractivas, que permiten comprender la articulada complejidad de las fronteras en Centroamérica, de las cuales Chiapas es parte.

Después, para complementar una panorámica regional, podrían revisarse dos aportaciones fundamentales, contenidas en el libro Chiapas. Los rumbos de otra historia (Viqueira \& Ruz, 2004). La primera, es un artículo de Juan Pedro Viqueira, "Chiapas y sus regiones", en la que el autor, sin proponerse caracterizar las regiones de la frontera, lo logra, al plantear una nueva división estatal, a partir de criterios sociales y culturales. Con base en datos lingüísticos, históricos y culturales, identifica, entre 
otras, seis regiones diferenciadas en la frontera México-Guatemala: Soconusco, Región de Motozintla (o Mariscal), Valle de Grijalva, Llanuras de Comitán y las Margaritas, La Selva Lacandona y Marqués de Comillas, útiles para delimitar áreas fronterizas de estudio. El segundo aporte es un conjunto de artículos, agrupados en el último apartado de libro, titulado: "La Selva Lacandona", donde se exponen, principalmente datos culturales e interpretaciones sociológicas del modo en que se desarrolló la región selvática, próxima a la frontera, previo al levantamiento del Ejército Zapatista de Liberación Nacional (EZLN), en 1994. De este bloque sobresalen dos capítulos: "De la sierra a la selva: identidades étnicas y religiosas en la frontera sur" (Hernández Castillo, 2004).

Otra obra imprescindible, de corte histórico, es la de Jan de Vos, quien, a través de fuentes de archivo, investigación etnográfica, testimonios e historias de vidas, logró mostrar la complejidad de la frontera. El autor sitúa la disputa regional, nacional e internacional al delimitarla, pero siempre aterrizado en sujetos particulares. Aunque su obra es vasta, de este autor se quieren destacar cuatro libros y dos artículos, principalmente. Por orden temático, el primero es Las fronteras de la frontera sur. Reseña de los proyectos de expansión que figuraron la frontera entre México y Centroamérica (De Vos, 1993). En este libro se pueden rastrear de manera sucinta cuatro proyectos históricos que se propusieron delimitar e instrumentar la frontera: el prehispánico, el inglés, el mexicano y el guatemalteco. Aquí se observará la intervención de distintas intencionalidades que condujeron a la delimitación actual de la frontera sur. La conformación de los límites territoriales quedará más esclarecida si se revisa el libro Oro verde. La conquista de la Selva Lacandona por los madereros tabasqueños (De Vos, 1996), que aborda las disputas entre empresas madereras en Tabasco, Chiapas y Guatemala, y cuya lucha influyó en el modo que se resolvieron los límites territoriales sobre el terreno a fines del siglo xix y principios del xx. Como paréntesis, en relación con este periodo, cabe revisar a la par dos textos asociados con el proceso de deslinde de la frontera México-Centroamérica. Uno es el de César Sepúlveda (1958): "Historia y problema de los límites de México: in la frontera sur", y el segundo, "La Comisión Mexicana de Límites y la definición de la frontera sur del país”, de Luz M. O. Tamayo (2015). En este último, la autora esclarece los retos y las decisiones que debió tomar dicha comisión para deslindar el territorio mexicano.

Otras recomendaciones de la obra de Jan de Vos son: Una tierra para sembrar sueños. Historia reciente de la Selva Lacandona 1950-2000 (De Vos, 2002b) y Viajes al desierto de la soledad. Un retrato hablado de la Selva Lacandona (De Vos, 2003), especializados en analizar extractos de viajeros que conocieron la Selva Lacandona y en testimonios de actores que la habitaron en la segunda mitad del siglo xix, y quienes relatan los pormenores de la dinámica social en esta región y su relación con Guatemala. Por último, se recomienda el texto: "Las fronteras de la frontera sur. Una visión histórica" (De Vos, 2002a), un clásico para conocer la dimensión política de este límite.

Otro investigador destacado es el antropólogo Andrés Fábregas, quien, a inicios de la década de 1980, lideró un proyecto sobre la frontera sur, adscrito al Centro de Investigaciones y Estudios Superiores en Antropología Social (CIESAS), pionero en su tipo. Fábregas ha dado cuenta de sus resultados en numerosos escritos, pero la mejor síntesis se encuentra en "La elaboración del concepto de frontera sur y la fundación del ciesas-Sureste. 1983-1985", incluido en el libro Marcos institucionales de la antropología en Chiapas a finales del segundo milenio (Fábregas, 2015, pp. 23-60). En este texto describe los estudios generados en el contexto del proyecto. Información que 
completa con las reseñas de estudios previos, de los cuales se nutrieron sus indagaciones. Entre ellos menciona la investigación de Juan Pohlenz sobre plantaciones de café en el Soconusco y su papel en el desarrollo de la economía de mercado, que dio pie a circuitos migratorios entre la población de Los Altos de Chiapas, el Soconusco y Guatemala. Dicho tema, señala Fábregas, sería más tarde profundizado en el contexto del proyecto de Mariano Baez y Patricia Ponce en el Soconusco.

Hay que hacer un nuevo paréntesis en este mismo bloque de estudios enfocados a exponer etnográficamente sobre cómo la economía cafetalera impactó en la conformación de la frontera en el Soconusco, para lo que se recomienda el estudio de Andrés Medina (1973), "Notas etnográficas sobre los mames de Chiapas", donde se muestra la estrecha relación entre la producción cafetalera y la conformación de poblados fronterizos mames, lo que abre una nueva veta de exploración antropológica, antes vista de refilón: la de la compleja diversidad étnica en el límite Chiapas-Guatemala.

Otro libro básico para comprender la trayectoria del sistema de regulación migratoria desprendida del trabajo jornalero en las plantaciones cafetaleras es el de Germán Martínez Velasco (1994), quien ofrece una detallada investigación sobre sus transformaciones. Su trabajo se titula Plantaciones, trabajo guatemalteco y política migratoria en la frontera sur de México. El argumento de este libro puede ser completado con el coordinado por Mercedes De Vega (2011), Historia de las relaciones internacionales de México 1821-2010. Necesario para entender la normativa sobre movilidad y migración en la frontera sur.

Fábregas (2015) señala que las variables identificadas por su equipo en 1983, y que hacían presente la frontera sur en el resto del país, eran tres: 1) la migración provocada por las guerras recientes en Centroamérica; 2) el descubrimiento de yacimientos de petróleo, y la posibilidad de expandir la exploración en el área para incentivar la búsqueda de hidrocarburos; y 3) la puesta en marcha de proyectos turísticos. Es curioso, sin embargo, que la continuidad que el ciesas dio a los estudios sobre la frontera no se basó en estos hallazgos sobre la migración forzada, la exploración extractiva, o el turismo, sino en otro aspecto, que a simple vista parece no ser tan apremiante: el estudio de la diversidad religiosa en el sur de México, gracias a lo cual se puede conocer, a través de estudios de caso, el papel de los distintos credos a lo largo de la frontera. De modo que el primer esfuerzo por entender la dinámica social en la frontera se cristalizó en cuatro tomos, publicados por el ciesas, sobre cambio religioso. Colección coordinada por el antropólogo Leonel Durán Solís.

De este interés del ciEsas por la frontera sur, primero dirigido por Andrés Fábregas y después por Durán Solís, sobresale el trabajo de la que entonces era una joven investigadora: Rosalva Aida Hernández Castillo (1988), quien escribió su tesis de licenciatura sobre la población kanjobal refugiada en México, a causa de la guerra en Guatemala, Mecanismos de reproducción social y cultural de los indígenas kanjobales refugiados en Chiapas. Y esta le abrió campo para dedicar décadas en desentrañar las complejas relaciones entre etnicidad, identidad, estado nacional, cambio religioso y organización comunitaria, en las dinámicas cotidianas entre Chiapas y Guatemala. Producto de sus observaciones publicó varios trabajos ahora fundamentales en el estudio de la frontera sur, tales como: "Los refugiados guatemaltecos y la dinámica fronteriza en Chiapas" (Hernández Castillo, 1992), La experiencia del refugio en Chiapas. Nuevas relaciones en la frontera sur mexicana (Hernández Castillo et al., 1993), "Identidades colectivas en los márgenes de la nación: etnicidad y cambio religioso entre los mames de Chiapas" (Hernández Castillo, 1994), La otra frontera. Identidades múltiples en el Chiapas poscolonial 
(Hernández Castillo, 2001) y Sur profundo. Identidades Indígenas en la frontera ChiapasGuatemala (Hernández Castillo, 2012).

No sobra decir que el refugio guatemalteco, provocado por el desplazamiento forzado, como consecuencia de la represión al movimiento armado en Guatemala, fue uno de los eventos de mayor incidencia para visibilizar la frontera sur a finales del siglo xx, por lo que una amplia bibliografía da cuenta del impacto que tuvo en la remarcación del límite fronterizo y en su resignificación. En ese contexto, para los mexicanos el deslindarse de los guatemaltecos fue un mecanismo de sobrevivencia entre la población regional para enfatizar su nacionalidad mexicana y evitar los ataques del ejército de Guatemala. Entre los trabajos que analizan este proceso se recomienda revisar: Los refugiados guatemaltecos en Campeche y Quintana Roo. Condiciones sociales y culturales de Sergio Aguayo y colaboradores (1989); Una década de refugio en México, coordinado por Graciela Freyermuth y Rosalva Aída Hernández Castillo (1992); Ser mexicano en Chiapas. Identidad y ciudadanización entre los refugiados guatemaltecos en La Trinitaria, Chiapas (Ruíz Lagier, 2013) y "Los refugiados guatemaltecos y la fronterafrente de discriminación, explotación y desigualdad" de Verónica Ruíz Lagier (2018). Respecto al tema destaca Edith Kauffer, quien cuenta también con una prolífica obra: Les réfugiés guatémaltèques au Chiapas. Le retour dupeuple du maïs. Un projet politique (2000a), "Refugiados Guatemaltecos en México: del refugio a la repatriación del retorno a la integración" (2000b), "De la frontera política a las fronteras étnicas: refugiados guatemaltecos en México" (2005), "Refugiados guatemaltecos y conformación de la frontera sur de Chiapas en los años ochenta" (1997), entre otros. Por su parte, Edith Kauffer profundizó, de forma posterior, en investigaciones sobre las normativas y disputas por las cuencas hidrográficas internacionales compartidas en esta región (2011a; 2011b; 2013).

Otra obra significativa que permite comprender la problemática dinámica de la frontera con Guatemala, en la década de 1980, es la de Ricardo Falla, quien aborda la compleja situación de las comunidades de población en resistencia del lado guatemalteco, en torno a la Franja Transversal del Norte. Es tan vasta su producción que recomendamos previamente revisar unas notas introductorias, redactadas por Enriqueta Lerma y Gerardo Monterrosa (2020). Su lectura permite identificar temáticas y seleccionar la bibliografía adecuada para comprender distintos aspectos de la frontera.

Otro eje, ya analizado, pero no agotado, es el papel de los grupos étnicos en la conformación de la frontera. Revisión ardua que rebasa estas líneas, pero que no podemos dejar fuera. Al tratar el tema, el investigador notará dos tipos de grupos que reproducen su cultura en torno a la frontera: aquellos que cuentan con un profundo arraigo histórico en la región (choles, tojolabales, jacaltecos, chicomultecos, mames y kanjobales), y los de asentamiento reciente, ya sea porque migraron en busca de tierras a partir de la década de 1960 (tsotsiles y tseltales), porque se expandieron con el refugio guatemalteco durante la década de 1980 (como chujes, kanjobales y acatecos) o porque recuperaron tierras con el levantamiento del EzLN en la última década del siglo xx. Contribuye a conocer la pluralidad del estado chiapaneco el libro coordinado por José del Val y colaboradores (2019): Estado del desarrollo económico y social de los pueblos indígenas de Chiapas, en el que se analizan estadísticamente y cualitativamente los procesos socioculturales y económicos del estado. Un libro básico de consulta en el que se encontrará material sobre la dinámica sociodemográfica de los pueblos de Chiapas, incluidos los de la región fronteriza, sus cosmovisiones y la diversidad de identidades. 
Desde una perspectiva histórica, se invita a leer el texto de Jan de Vos (1994), Vivir en frontera. La experiencia de los pueblos de Chiapas, el cual no versa específicamente sobre lo que ahora entendemos como frontera límite (sistema de contención), sino como frontera frente (avanzada o expansión). En este trabajo es posible conocer el modo en que se amplió la frontera colonial y su proceso de producción hasta el periodo de la revolución mexicana. Este enfatiza en los pueblos indígenas, tanto oriundos como allegados, describe sus encuentros y disputas en el proceso de conformación política de la región. Otras obras importantes en este asunto son las de Ruth Piedrasanta Herrera (2009): Los chuj. Unidad y rupturas en su espacio; Historia chuj a contrapelo. Huellas de un pueblo con memoria de Fernando Limón (2009); y Voces de la historia. Nuevo San Juan Chamula, Nuevo Huixtán, Nuevo Mazatán, de Ana María Garza y colaboradores (1994). Asimismo, para adentrarse al caso mam, un pueblo maya más atravesado por la frontera, se recomienda leer: "Los mam de México y Guatemala: un pueblo binacional entre la autonomía y la heteronomía”, de Miguel Ángel Toledo-Pineda y Enrique Coraza de los Santos (2019).

De suma importancia para comprender las estrategias usadas a fines del siglo $\mathrm{xx}$ con el objetivo de reordenar la frontera con Guatemala es revisar el contexto que antecede a estas decisiones. Conviene no perder de vista que se trata de un espacio de marginalidad étnico-campesino. Por ello se recomienda revisar el libro en el que participan varios autores, coordinado por David Moctezuma Navarro: Chiapas. Los problemas de fondo (1994); y el de Xóchitl Leyva y Gabriel Ascencio (2002), Lacandonia al filo de agua. Aunque los textos se dedican a exponer las casusas del levantamiento del EzLN, la frontera aparece de manera constante en sus análisis. Estos textos se completan con la revisión del proceso de remunicipalización que atravesó Chiapas tras la insurrección de la guerrilla zapatista y el modo en que esto configuró un nuevo ordenamiento territorial y una nueva forma de administrar la frontera sur. Destacan, los trabajos realizados por Sonia Toledo, Luis Rodríguez, Neil Harvey, María del Carmen Solís y Jesús Solís, agrupados en una obra editada por Xóchitl Leyva y Araceli Burguete (2007), y nutrida con sus propias aportaciones: Remunicipalization in Chipas. Politics and the political in times of counter-insurgency. Del mismo tipo se puede encontrar: Estudios monográficos. Nuevos municipios en Chiapas - en dos volúmenes-, coordinado por las mismas autoras (Leyva \& Burguete, 2004) y Maravilla Tenejapa. Monografia municipal de Luis Rodríguez (2017).

Son numerosos los resultados de investigación sobre el tema publicados en lo que va del siglo xxi. Muchos ya mencionados más arriba. Abordar la bibliografía existente sería excesivo, por lo que se delimita la búsqueda a aquella que estudia el modo en que se configura la frontera a partir de quienes en ella reproducen su vida cotidiana. En ese sentido, la consulta se refiere, en primera instancia, a libros de autoría individual. Se destacan aquí tres obras: Frontera Sur Chiapaneca. El muro de la violencia. Análisis de la normalización de la violencia hacia los migrantes indocumentados en tránsito de Soledad Álvarez (2016), un libro fascinante, en que se expone etnográficamente la cotidianidad de quienes viven la frontera sur, próxima a Ciudad Hidalgo, y su continuidad en Tapachula. La autora describe, a partir de descripciones puntuales, la experiencia que viven en este espacio los traileros, las trabajadoras de centros nocturnos, el personal de centros migrantes, el Instituto Nacional de Migración, las trabajadoras domésticas, los vendedores ambulantes, los niños que laboran en los parques, entre otros sujetos sociales, que han hecho de la frontera su forma de subsistencia. Desde la perspectiva de la vida cotidiana y su vinculación con el Estado sobresale el artículo "Configuración 
regional del Estado: orden mercantil y comunidad interpretativa en la frontera MéxicoGuatemala”, de Hugo Rojas Pérez y Héctor Fletes Ocón (2017), en el que se describen las dinámicas que se contruyen alrededor de la venta transfronteriza de verduras, sobre el río Suchiate, y cuya práctica se reproduce a contrapelo de la normatividad estatal, generando un espacio de permisividad fronterizo.

Asimismo, se recomienda el libro de Carmen Fernández (2017), La vida en una orilla del sur. Inmigración hondureña en dos ciudades de la frontera Chiapas-Guatemala. La obra profundiza en las formas de apropiación del espacio en el Soconusco por inmigrantes hondureños y de sus redes sociales, articuladas para enfrentar los retos que impone a esta población su condición de avecinados y sus anhelos por trasladarse más al norte del continente. Textos que bien pueden completar este tema, desde una perspectiva de género, son los de Dulce K. Ramírez López (2018), Mujeres migrantes en la frontera sur de México: aproximaciones desde la insterseccionalidad y el de Rojas Wiesner y Ángeles Cruz (2012), "La situación de las mujeres migrantes en la frontera de México con Guatemala". Con estas publicaciones es posible acercarse a la dinámica social de los inmigrantes centroamericanos asentados en la región de interés.

Por último, para completar el estudio reciente de la dinámica social en la frontera, se concluye con dos libros, el de Enriqueta Lerma (2019b): Los otros creyentes. Territorio y tepraxis de la Iglesia liberadora en la región fronteriza de Chiapas, en el que la autora aborda los problemas que se experimentan en lo que ella llama "la frontera intermedia ChiapasGuatemala”. Analiza el empeño de la feligresía de la iglesia liberadora (teología de la liberación) por intervenir en el modo en que se organiza el territorio en la frontera sur, principalmente en los municipios de Frontera Comalapa y Chicomuselo, donde los creyentes se interesan en descriminalizar la migración centroamericana y en frenar el extractivismo minero que amenaza su territorio. El segundo libro, el de Mario Valdez (2006), Desencuentro y encuentro de fronteras: el Petén guatemalteco y el sureste mexicano 18951949, aborda, desde la perspectiva de la historia regional, la forma en que el territorio fronterizo fue apropiado en el contexto de inserción de la selva al sistema productivo global. Así, el autor, para explicar la construcción de un espacio regional transfronterizo, analiza la inversión económica, los proyectos extractivos y las disputas entre distintas empresas, que configuraron el desarrollo de la actual frontera.

Por otra parte, se encuentra una serie de libros colectivos que abordan distintos aspectos sociales, históricos, de género, laborales, de salud y migratorios, tales como: Identidades, migraciones y género en la frontera sur de México, coordinado por Edith Kauffer (2002). El coordinado por Hugo Ángeles Cruz y colaboradores (2005): Actores y realidades en la frontera sur de México. El coordinado por Carolina Rivera (2014), Trabajo y vida cotidiana de centroamericanos en la frontera suroccidental de México, en el que se analizan las condiciones laborales de los migrantes guatemaltecos, salvadoreños y hondureños que decidieron permanecer en la región del Soconusco. Entre estas obras colectivas se cuenta con dos textos: uno escrito desde una mirada macro, coordinado por Daniel Villafuerte Solís (2004): La Frontera sur de México. Del TLC México-Centroamérica al Plan Puebla-Panamá, y el otro con una perspectiva más acotada, que pone énfasis en las personas y objetos que cruzan la frontera: Regímenes de movilidad en la frontera México-Guatemala: gobernanza transfronteriza para el desarrollo, de María del Rocío Barajas Escamilla y colaboradores (2021).

Mención aparte merece el libro colectivo Vidas transfronterizadas: dinámicas y actores en el límite Guatemala/México, siglo XIX-XXI, coordinado por Justus Fenner y colaboradores (2020), producto de un grupo de investigación binacional México-Guatemala. El texto 
aborda el proceso de producción histórica de esta frontera, con un ingrediente que no existió en las obras reseñadas hasta ahora, y que es la participación de investigadores de ambos lados del límite, por lo que el texto contempla ambas miradas en un periodo histórico amplio y con diversas aristas.

Sobre la forma en que se percibe esta frontera hay resultados de investigación en artículos y capítulos de libro. Se recomiendan: “'Guatemalteco-mexicanoestadounidenses' en Chiapas: familias con estatus ciudadano diferenciado y su multiterritorialidad" (Lerma, 2016), donde la autora muestra el modo en que el territorio es percibido de manera diversa, según las posibilidades de movilidad de un grupo de origen guatemalteco asentado en un municipio fronterizo. El artículo: "La línea '...está ahí, es algo que se ve, pero que también se siente': imaginarios de frontera de las juventudes 'al sur'" de Amalia Campos Delgado (2010), donde se expone el modo en que es percibido el límite fronterizo por jóvenes que se han planteado el reto de pasar dos fronteras, la México-Guatemala y la México-Estados Unidos. También conviene revisar el estudio de Diego Noel Ramos Rojas (2020), "La triple frontera: propuesta conceptual para explicar las dinámicas de la región fronteriza entre México y Guatemala", en el que se exponen las distintas percepciones que sobre el espacio mantienen distintos actores migrantes, según su nacionalidad.

Un rubro distinto, con un enfoque más geopolítico, se agrupa en el siguiente apartado.

\section{Sobre el ordenamiento territorial y la lógica de la seguridad nacional: textos y documentos}

Como se dijo líneas arriba, Andrés Fábregas (2015) identificó la inauguración de un proceso de control migratorio en la frontera sur en la década de 1980. Este se vio consolidado con la intervención de organismos nacionales e internacionales, dedicados a identificar, auxiliar y regular el paso de centroamericanos a México, especialmente guatemaltecos, que llegaron a México, resultado de los conflictos armados en la región. Una década después, las intenciones de reordenar el territorio fronterizo serían resultado de acuerdos tomados durante la firma del Tratado de Libre Comercio.

María del Carmen García identifica el fortalecimiento de los dispositivos de control fronterizo como parte de acuerdos internacionales:

la subordinación de México a la agenda de seguridad del gobierno estadounidense alcanza su expresión máxima en las administraciones de Vicente Fox y Felipe Calderón, y puede ser ejemplificada con el tratamiento dado al fenómeno de la migración irregular de tránsito. (García, 2015, p. 60)

Bajo esta tesitura, en este apartado se mencionan las pistas bibliográficas y documentales a seguir a partir de que se nutrió el discurso de reordenar el territorio del sureste —fincado en el desarrollo regional— y el objetivo de "garantizar la seguridad nacional fronteriza".

Fue a inicios del siglo xxi que la frontera sur experimentó estrategias decididas a producir una espacialidad controlada. En esta tarea tuvo dominio un conjunto de medidas económicas, destinadas a reordenar el territorio de manera productiva, y la celebración de acuerdos, convenios y planes, para hacerla "segura". Sobre estos puntos se cuenta con algunas publicaciones especializadas que facilitan la indagación. En la 
última década del siglo xix, Rodolfo Casillas identificó ciertas claves, dadas a conocer en el texto "Migraciones centroamericanas en México. Semblanza de un proceso social emergente" (Casillas, 1991). En su artículo sugirió poner atención a la diversidad de los flujos migratorios que cruzan la frontera sur y a las distintas formas en que los allegados permanecían legal o ilegalmente en el área, ya sea como trabajadores temporales, refugiados, desplazados o transmigrantes. Casillas encuentra en el intento de registrar, controlar y disminuir estos flujos migratorios diversos, el reforzamiento de una política de acoplamiento a las políticas migratorias estadounidenses.

En esta misma línea se recomienda revisar los resultados de César E. Ordoñez (1994) en Modernización y desarrollo regional en Chiapas. Un caso: la zona libre de Tapachula y "Tendencias de la integración económica fronteriza en Chiapas y Guatemala" (Ordoñez, 2001), estudios en los que expone la forma en que se ha planeado el desarrollo del sur de México y Centroamérica como parte de una estrategia de integración económica, que incluye hacer "productiva" la frontera sur de México. Asimismo, cabe revisar: "Las fronteras de la Frontera sur" de Daniel Villafuerte y María del Carmen García (2005), "La migración irregular de tránsito desde el derecho y la política en el tiempo global. El caso de México" de María del Carmen García (2015), y el ensayo de Úrsula Roldán, "La frontera estratégica y extendida, MéxicoCentroamérica. Sus impactos: 'Seguridad nacional versus derechos humanos de la población migrante"” (2017). Estos estudios permiten conocer, de manera puntual y cronológica, las disposiciones político-económicas aplicadas a esta frontera, al tomar en cuenta el impacto de las disposiciones de la economía global en lo local.

Por otra parte, dos artículos ofrecen una mirada sociológica del proceso de producción imaginaria de las fronteras internacionales contemporáneas, con énfasis en la instrumentación política de la frontera Chiapas-Guatemala: "Esta orilla que es nuestro centro. Producción imaginaria de la frontera: Una mirada desde el borde Chiapas-Guatemala", de Enriqueta Lerma (2019a), y "La producción de una franja fronteriza controlada. El ordenamiento territorial en el sureste mexicano", de Fenner y colaboradores (2020).

Se considera que con esta bibliografía es posible introduir y dar seguimiento a la compleja relación política en las distintas escalas global, nacional y regional, y a los distintos instrumentos que se han desarrollado y se aplican en el control fronterizo México-Guatemala. Del mismo modo, otras fuentes a las que se puede recurrir de manera directa son las minutas e informes anuales publicados por la Comisión de Asuntos Fronterizos Sur del Senado de la República, ubicadas en su portal de internet (Comisión de Asuntos Fronterizos Sur Lxin legislatura 2016; Comisión de Asuntos Fronterizos Sur LXII-LXIII legislaturas, 2018). Esta página registra, en dos décadas, al menos ocho iniciativas dirigidas a impulsar el desarrollo económico en la región. Se mencionan aquí con el fin de ofrecer una especie de listado que puede ser de interés: el Plan Puebla Panamá en el año 2000, el Fideicomiso para el Desarrollo Regional del Sur Sureste en 2001, la Comisión para el Desarrollo Integral de la Región SurSureste en 2004, y el Proyecto de Integración y Desarrollo de Mesoamérica en 2008. En 2014, la comisión registra el impulso de dos fondos: el Fondo para la Frontera y el Fondo Sur-Sureste, ambos para realizar estudios y proyectos. Para 2016 se menciona la readecuación de dos iniciativas: el Fondo para la Competitividad de la Frontera y la puesta en marcha del Programa Regional de Desarrollo Sur-Sureste, dirigido a impulsar programas productivos. 
También es de consultar la actualización que se hizo a la normativa sobre migración laboral y de corta estancia, realizada durante el gobierno de Felipe Calderón. Así como el Plan de Reordenamiento de la Frontera Sur, de 2008, con el que se reguló la expedición de permisos a guatemaltecos y beliceños. De este mismo año, se recomienda revisar la primera Carta de Acuerdo sobre la Iniciativa Mérida, firmada entre México y Estados Unidos, y que implicó la cooperación y reconocimiento de responsabilidades compartidas para contrarrestar la violencia que el tráfico de drogas provoca en ambos lados de la frontera. Es de resaltar los cuatro pilares acordados en la Iniciativa Mérida: 1. afectar la capacidad operativa del crimen organizado; 2. institucionalizar la capacidad para mantener el Estado de derecho; 3. crear la estructura fronteriza del siglo xxI; y, 4. construir comunidades fuertes y resilientes (Embajada y Consulados de Estados Unidos en México, s. f.). Estos pilares instrumentan una frontera con mayores dispositivos de control migratorio.

La Iniciativa Mérida representó un paso contundente en el avance de la frontera externa de Estados Unidos en territorio mexicano. Con ella se incentivó en 2008 la criminalización de los espacios fronterizos. Lo que supuso el tránsito de una política interesada en el desarrollo económico por otra ajustada a la seguridad nacional; lógica que cobró singular importancia en el diseño del actual ordenamiento territorial fronterizo, donde ocupa un lugar relevante el Programa Frontera Sur.

En esta misma tesitura, es de suma importancia poner atención al modo en que se instrumentó el programa mencionado, punta del iceberg de un conjunto de medidas vinculadas a la seguridad nacional, y que representó la continuidad del Programa de Seguridad Nacional 2014-2018 (DOF, 2014b). Con este segundo programa se transformó el concepto de seguridad que se usaba en el diagnóstico de la frontera sur. Se propuso un nuevo abordaje multidimensional que incluyó la vigilancia del sistema de salud, del nutricional, del ecológico, del laboral, entre otros, dispuesto a mantener la seguridad de la soberanía nacional. A partir de esta modificación se priorizó la "prevención", por lo que el documento dedica una parte importante a explicar la necesidad de detectar las posibles amenazas que pueden "obstaculizar la seguridad", y delega al poder Ejecutivo la toma de decisiones en esta materia, con la capacidad de "disponer de la totalidad de la fuerza armada permanente, es decir del Ejército, de la Armada y de la Fuerza Aérea, para garantizar la seguridad interior y defensa exterior de la federación” (DOF, 2014b), incluida, especialmente, la frontera sur.

El protagonismo del Programa Frontera Sur, impulsado por el presidente Enrique Peña Nieto en 2014, reside en que, aunque se justificó como parte de un plan para desarrollar económicamente la región, en realidad parece tener otros objetivos, ya que se ha hecho cargo de resguardar la seguridad fronteriza. Destaca el interés por concluir la construcción de Centros de Atención Integral al Tránsito Fronterizo (CAITF), cuyo objetivo programático inicial era vigilar el comercio exterior y mejorar el tránsito carretero, ${ }^{2}$ según lo publicado en el Diario Oficial de la Federación (DOF, 2014a). No obstante, lo que se observa en el terreno es que los CAITF se dedican a detener migrantes y a deportarlos; además de fungir como barreras a la movilidad regional y ser marcadores del territorio fronterizo.

\footnotetext{
${ }^{2}$ Las dependencias que conforman el Centro de Atención al Transito Fronterizo son Secretaría de Gobernación, Secretaría de la Defensa Nacional, Secretaría de Marina, Secretaría de Hacienda y Crédito Público, Secretaría de Agricultura, Ganadería, Desarrollo Rural, Pesca y Alimentación y Secretaría de Salud.
} 
Además de poner atención a este programa, se sugiere profundizar en el incremento de la militarización en el área. Como ejemplo, cabe señalar la inauguración del cuartel del Batallón de Infantería 101 con 600 elementos en Chicomuselo. El cual, declaró en su momento, Manuel Velasco, gobernador de Chiapas, se encargaría de fortalecer las acciones y protocolos de seguridad en la región y en la frontera sur (Coutiño, 2018, p. 23). El cuartel se suma a otras estaciones militares, previamente instaladas: tres de la Compañía de Infantería No Encuadrada (CINE) en Maravilla Tenejapa, Boca Lacantum y Campamento Lacantum; dos bases militares en Puerto Chiapas y Motozintla, y el $4^{\circ}$ Regimiento de Caballería Motorizada en Tapachula. Además de dos bases militares en Chiapas, una en Comitán y otra en Tuxtla Gutiérrez. A esta última se añadió el Escuadrón de Detección y Alerta Temprana, en 1987, para el resguardo de la frontera sur (Comisión de Defensa Nacional LXII legislatura Cámara de Diputados \& Secretaría de la Defensa Nacional, 2014). Con estos datos se puede pensar en el papel que cumple el ejército en el resguardo de la frontera, el cual no debe quedar al margen de las indagaciones.

Para profundizar en el ámbito de la planificación territorial se recomienda revisar el artículo "La producción de una franja fronteriza controlada. El ordenamiento territorial en el sureste mexicano" de Enriqueta Lerma (2020), en el que se muestra el intento de potencializar económicamente la región con el interés de contener la migración centromericana, a través del fortalecimiento de áreas productivas y el desarrollo de vías de comunicación, en aras de consolidar un circuito migratorio.

\section{Para concluir}

Con lo reseñado arriba no es posible argumentar que se está ante una frontera por descubrir o de la que se tengan pocos datos. Al contrario: el análisis de la frontera sur, Chiapas-Guatemala, cuenta ya con un cuerpo considerable de resultados de investigación, al que constantemente se suman nuevos títulos. La revisión bibliográfica hizo posible mostrar la diversidad de temas y enfoques, así como las problemáticas que se han analizado en el intento de hacer más comprensible este espacio. Pero también permitió encontrar aspectos comunes, investigaciones en ciernes y temas emergentes o por explorar. Entre otras cuestiones, es de destacar que, en distinción con los trabajos realizados previos al siglo XXI, actualmente los textos evitan desarrollar explicaciones exhaustivas sobre la historia de la delimitación internacional de la frontera y procuran no extenderse en descripciones puntuales sobre geografía o demografía. Al contrario, como parte de los avances de investigación se abordan los objetivos de estudio desde las primeras páginas y se remite al estado del arte para contextualizar la discusión central.

Hay otros aspectos transversales presentes en la mayor parte de los trabajos. Permanece el interés por visibilizar la diversidad étnica en la frontera, conformada por población originaria mayense (chuj, mame, kanjobal, acateca, tojolabal, tsotsil y tseltal), con el objetivo de subrayar sus características específicas y su continuidad histórica. Esta mirada permite situar las continuidades, pero también repensar y complejizar los análisis de frente a la heterogeneidad social reciente, resultado de nuevos flujos migratorios (centroamericanos, africanos y caribeños).

Asimismo, permanece la aproximación al estudio de la frontera, y la considera no como un territorio homogéneo sino como un área constituida por diversas regiones 
ecosistémicas, históricamente complejizadas por distintos planes de desarrollo, diversas actividades productivas y sucesivas oleadas de colonización. A este enfoque se añade el análisis de procesos económicos y políticos locales, regionales y globales recientes, y su impacto en distintos tramos de la frontera. Si bien, no se trata de análisis innovadores, cobran nuevos sentidos al tratar la instrumentación de la frontera a partir de acuerdos políticos nacionales e internaciones sobre seguridad fronteriza y al analizar las secuelas diferenciadas a lo largo del límite internacional.

Destaca, por otra parte, el incremento de los estudios migratorios en esta frontera, mismos que transitaron, en un primer momento, del analizar la movilidad regional en circuitos pendulares, jornaleros y temporales mexicano-guatemaltecos, hacia un espectro más amplio del fenómeno, con énfasis en la migración centroamericana. Esto ha conducido a no ver más esta frontera solo como un límite binacional (MéxicoGuatemala) o trinacional (México-Guatemala-Belice), sino como un espacio al que se conectan otros procesos fronterizos.

Tal como pronosticó Andrés Fábregas (2015, p. 32) en la década de 1980, efectivamente ha tomado mayor trascendencia el interés por tres temas: el primero, la migración, a la que ya se ha hecho referencia; el segundo, la exploración y explotación de yacimientos de hidrocarburos y de minerales en la frontera sur, así como los conflictos sociales derivados de estas actividades; y, en tercer lugar, el desarrollo del turismo en áreas naturales protegidas, como espacios regulados que impiden la proliferación de asentamientos humanos.

Otros temas, en cambio, han sido menos trabajados; entre estos, la percepción que los propios habitantes de la frontera mantienen sobre las dinámicas sociales que los atraviesan y el modo en que sus medios de vida se han transformado con las intervenciones securitivas y por la propia migración a otras partes de México y de Estados Unidos. También se reciente la poca producción de estudios con perspectiva de género. Por el contrario, sobresale el incremento de pesquisas sobre políticas de securitización y violencia, derechos humanos, y estudios exploratorios que dan cuenta del incremento de la migración infantil y de las caravanas masivas de migrantes centroamericanos.

Sin duda, la bibliografía que se revisará a la brevedad tocará otros temas, actualmente en desarrollo. Entre estos, el impacto social de la Guardia Nacional en los distintos tramos fronterizos, el efecto social y político de la migración haitiana en el Soconusco, engrosada entre 2020 y 2021, y en el último año las secuelas sociales que acarrearon las restricciones provocadas por la pandemia del COVID-19.

Por el momento, se espera que lo expuesto en este documento resulte de interés para conocer el estado de la cuestión y permita identificar vacíos que habrán de trabajarse a detalle. Los estudios aquí expuestos no agotan lo que hasta el momento se ha escrito. Aun así, se espera que este documento brinde direccionalidad a quienes se interesen por analizar los procesos que concurren en la frontera sur, especialmente en el tramo Chiapas-Guatemala.

\section{Agradecimientos}

Esta contribución forma parte del Programa de Apoyo a Proyectos de Investigación e Innovación Tecnológica, PAPIIT IN300620, promoción 2020, de la Universidad Nacional Autónoma de México. 


\section{Referencias}

Aguayo, S., Christensen, H., O’Dogherty Madrazo, L. \& Varesse, S. (1989). Los refugiados guatemaltecos en Campeche y Quintana Roo. Condiciones sociales y culturales. Instituto de Investigaciones de las Naciones Unidas para el Desarrollo Social/ El Colegio de México.

Álvarez, S. (2016). Frontera sur chiapaneca. El muro de la violencia. Análisis de la normalización de la violencia hacia los migrantes indocumentados en tránsito. Universidad Iberoamericana.

Ángeles Cruz, H. M. \& Huicochea Gómez, L., Saldívar Moreno, A. \& Tuñón Pablos, E. (Coords.). (2005). Actores y realidades en la frontera sur de México. Conapo/El Colegio de la Frontera Sur.

Barajas Escamilla, M. R., Ortiz Gómez, M. G. \& Kosigyna, L. (2021). Regímenes de movilidad en la frontera México-Guatemala. Gobernanza transfronteriza para el desarrollo. CIESAS/ Centro Geo/Cide/Instituto Mora/El Colegio de la Frontera Sur.

Bovin, P. (1997). Las fronteras del Istmo. Fronteras y sociedades entre el sur de México y América Central. Centro de Investigaciones y Estudios Superiores en Antropología Social/Centro de Estudios Mexicanos y Centroamericanos.

Campos Delgado, A. E. (2010). La línea “...está ahí, es algo que se ve, pero que también se siente": imaginarios de frontera de lasjuventudes "al sur". LiminaR, 8(2), 46-70. http:/ / www.scielo.org.mx/scielo.php?script=sci_arttext\&pid=S1665-80272010000200004\&l$\mathrm{ng}=\mathrm{es} \&$ tlng=es

Casillas, R. (1991). Migraciones centroamericanas en México. Semblanza de un proceso social emergente. Relaciones, 47, 67-81. https://www.colmich.edu.mx/relaciones25/files/revistas/046/RodolfoCasillasR.pdf

Comisión de Asuntos Fronterizos Sur LXII-LXIII legislaturas. (2018). Memoria de labores de comisiones. Cámara de Senadores. http://www.senado.gob.mx/comisiones/ asuntos_fronterizos_sur/docs/MEMORIA_CO_Asuntos_Fronterizos_Sur.pdf

Comisión de Asuntos Fronterizos Sur Lxiı legislatura. (2016). Programa de trabajo 2016. Cámara de Senadores. http://www.senado.gob.mx/comisiones/asuntos_ fronterizos_sur/docs/Programa1_LXIII.pdf

Comisión de Defensa Nacional Lxir legislatura Cámara de Diputados \& Secretaría de la Defensa Nacional. (2014). Fuerza Aérea Mexicana. La aviación militar, un siglo de historia (1915-2015). ALDvs. http://biblioteca.diputados.gob.mx/janium/bv/ md/LXII/FAM_aviacion_militar.pdf

Coutiño, G. (2018, 9 de diciembre). Inauguran cuartel militar en Chicomuselo. Meridiano 90.

De Vega, M. (2011). Historia de las relaciones internacionales de México 1821-2010 (vol. 2, Centroamérica). Secretaría de Relaciones Exteriores.

De Vos, J. (1993). Las fronteras de la frontera sur. Reseña de los proyectos de expansión que figuraron la frontera entre México y Centroamérica. Universidad Juárez Autónoma de Tabasco.

De Vos, J. (1994). Vivir en frontera. La experiencia de los pueblos de Chiapas, México. Centro de Investigaciones y Estudios Superiores en Antropología Social.

De Vos, J. (1996). Oro verde. La conquista de la selva Lacandona por los madereros tabasqueños. Fondo de Cultura Económica/Instituto de Cultura de Tabasco. 
De Vos, J. (2002a). Las fronteras de la frontera sur. Una visión histórica. En E. F. Kauffer Michel (Ed.), Identidades, migraciones y género en la frontera sur de México (pp. 49-67). El Colegio de la Frontera Sur.

De Vos, J. (2002b). Una tierra para sembrar sueños. Historia reciente de la selva Lacandona 1950-2000. Fondo de Cultura Económica/Centro de Investigaciones y Estudios Superiores en Antropología Social.

De Vos, J. (2003). Viajes al desierto de la soledad. Un retrato hablado de la selva Lacandona. Centro de Investigaciones y Estudios Superiores en Antropología Social/Miguel Ángel Porrúa.

Del Val, J., Sánchez, C. \& Zolla, C. (Coords.). (2019). Estado del desarrollo económico y social de los pueblos indígenas de Chiapas. Programa Universitario de Estudios de la Diversidad Cultural Interculturalidad-Universidad Nacional Autónoma de México.

Diario Oficial de la Federación (DOF). (2014a). Acuerdo por el que se instruye la constitución de los Centros de Atención Integral al Tránsito Fronteriza (CAITF). https://www. dof.gob.mx/nota_detalle.php?codigo $=5351464 \&$ fecha $=08 / 07 / 2014$

Diario Oficial de la Federación (DOF). (2014b). Programa de Seguridad Nacional 2014-2018, 30 de abril. http://www.dof.gob.mx/nota_detalle.php?codigo=5342824\&fecha $=30 / 04 / 2014$

Embajada y Consulados de Estados Unidos en México. (s. f.). Iniciativa Mérida. https:// mx.usembassy.gov/es/tag/iniciativa-merida/

Fábregas, A. (2015). Marcos institucionales de la antropología en Chiapas a finales del segundo milenio. Centro de Estudios Superiores de México y Centroamérica-Universidad de Ciencias y Artes de Chiapas.

Fenner, J., Lerma Rodríguez, E., Piedrasanta Herrera, R. \& Torras Conangla, R. (Coords.). (2020). Vidas transfronterizadas: dinámicas y actores en el límite Guatemala/ México, siglo XIX-XXI. Centro de Investigacioes Multidisciplinarias sobre Chiapas y la Frontera Sur-unAM.

Fernández, C. (2017). La vida en una orilla del sur. Inmigración hondureña en dos ciudades de la frontera Chiapas-Guatemala. Centro de Investigaciones y Estudios Superiores en Antropología Social-Sureste.

Freyermuth, G. \& Hernández, R. (1992). Una década de refugio en México. Centro de Investigaciones y Estudios Superiores en Antropología Social-Sureste/Academia Mexicana de Derechos Humanos.

García, M. C. (2015). La migración irregular de tránsito desde el derecho y la política en el tiempo global. El caso de México. En M. E. Anguiano \& D. Villafuerte (Coords.), Cruces de fronteras. Movilidad humana y políticas migratorias (pp. 5281). El Colegio de la Frontera Norte/Centro de Estudios Superiores de México y Centroamérica/Universidad de Ciencias y Artes de Chiapas. https://colef. repositorioinstitucional.mx/jspui/bitstream/1014/544/1/Cruces\%20de\%20 fronteras_Int.pdf

Garza, A. M. \& Paz, M. F., Ruiz, J. M. \& Calvo, A. (1994). Voces de la historia. Nuevo San Juan Chamula, Nuevo Huixtán, Nuevo Mazatán. Centro Regional de Investigaciones Multidisciplinarias-Universidad Nacional Autónoma de México. 
Guerrero, A. (2007). Imágenes de América Latina y México a través de los mapas mentales. En A. Arruda \& M. Alba (Eds.), Espacios imaginarios y representaciones sociales (pp. 235-285). Anthropos/Universidad Autónoma Metropolitana-Iztapalapa.

Hernández Castillo, R. (1988). Mecanismos de reproducción social y cultural de los indígenas kanjobales refugiados en Chiapas [Tesis de licenciatura, Escuela Nacional de Antropología e Historia].

Hernández Castillo, R. (1992). Los refugiados guatemaltecos y la dinámica fronteriza en Chiapas. En G. Freyermuth \& R. Hernández (Eds.), Una década de refugio en México (pp. 93-105). Centro de Investigaciones y Estudios Superiores en Antropología Social/Instituto Chiapaneco de Cultura/Academia de Derechos Humanos.

Hernández Castillo, R. (1994). Identidades colectivas en los márgenes de la nación: etnicidad y cambio religioso entre los mames de Chiapas. Nueva Antropología, 13(45), 83-105.

Hernández Castillo, R. (2001). La otra frontera. Identidades múltiples en el Chiapas poscolonial. Ciesas-Miguel Ángel Porrúa.

Hernández Castillo, R. (2004). De la sierra a la selva: identidades étnicas y religiosas en la frontera sur. En J. P. Viqueira \& M. H. Ruz (Eds.), Chiapas. Los rumbos de otra historia. Centro de Investigaciones y Estudios Superiores en Antropología Social/Universidad Nacional Autónoma de México.

Hernández Castillo, R. (2012). Sur profundo. Identidades indígenas en la frontera Chiapas-Guatemala. Centro de Investigaciones y Estudios Superiores en Antropología Social/Comisión Nacional de Desarrollo de los Pueblos Indígenas.

Hernández Castillo, R., Nava Zamora, N., Flores Arenales, C. \& Escalona Victoria, J. L. (1993). La experiencia del refugio en Chiapas. Nuevas relaciones en la frontera sur mexicana. Academia Mexicana de Derechos Humanos.

Kauffer, E. F. (1997). Refugiados guatemaltecos y conformación de la frontera sur de Chiapas en los años ochenta. En P. Bovin (Dir.), Las fronteras del Istmo. Fronteras y sociedades entre el sur de México y América Central (pp. 163-170). Centro de Investigaciones y Estudios Superiores en Antropología Social/Centro Francés de Estudios Mexicanos y Centroamericanos.

Kauffer, E. F. (2000a). Les réfugiés guatémaltèques au Chiapas. Le retour du peuple du maïs. Un projet politique (Collection Logiques Politiques). L'Harmattan.

Kauffer, E. F. (2000b). Refugiados guatemaltecos en México: del refugio a la repatriación del retorno a la integración. Boletín Conapo, 4(12), 7-12. https://www. academia.edu/34411955/Refugiados_guatemaltecos_en_M\%C3\%A9xico_del_ refugio_a_la_repatriaci\%C3\%B3n_del_retorno_a_la_integraci\%C3\%B3n_Bolet\%C3\%ADn_de_Migraci\%C3\%B3n_Internacional_12_CONAPO_p_7_12

Kauffer, E. F. (Ed.). (2002). Identidades, migraciones y género en la frontera sur de México. El Colegio de la Frontera Sur.

Kauffer, E. F. (2005). De la frontera política a las fronteras étnicas: refugiados guatemaltecos en México. Frontera Norte, 17(34), 7-36. http://www.scielo.org.mx/ scielo.php?script=sci_arttext\&pid=S0187-73722005000200001\&lng=es\&tlng=es

Kauffer, E. F. (Coord.). (2011a). Entre manantiales y ríos desatados: paradojas de las hidropolíticas fronterizas (México-Guatemala). ciesas/Colmich. 
Kauffer, E. F. (2011b). Hidropolíticas en la frontera entre México, Guatemala y Belice: la necesaria redefinición de un concepto para analizar la complejidad de las relaciones en torno al agua en escenarios transfronterizos. Aqua-Lac, 3(1), 157166. http://aqua-lac.org/index.php/Aqua-LAC/article/view/81/58

Kauffer, E. F. (2013). De las indefiniciones a las demarcaciones inacabadas: repensar las fronteras fluviales y terrestres entre México, Guatemala y Belice. Liminar. Estudios sociales y humanisticos, 11(2), 70-81. https://doi.org/10.29043/liminar. v11i2.223

Lerma, E. (2016). "Guatemalteco-mexicano-estadounidenses" en Chiapas: familias con estatus ciudadano diferenciado y su multiterritorialidad. Migraciones Internacionales, 8(30), 95-124. https://doi.org/10.17428/rmi.v8i3.616

Lerma, E. (2019a). Esta orilla que es nuestro centro. Producción imaginaria de la frontera: Una mirada desde el borde Chiapas-Guatemala. Espacios políticos, 11(18), 21-38. https://www.academia.edu/41126164/Esta_orilla_que_es_nuestro_centro._Producci\%C3\%B3n_imaginaria_de_la_frontera_Una_mirada_desde_el_ borde_Chiapas-Guatemala

Lerma, E. (2019b). Los otros creyentes. Territorio y tepraxis de la Iglesia liberadora en la región fronteriza de Chiapas. Centro de Investigaciones Multidisciplinarias sobre Chiapas y la Frontera Sur/unam.

Lerma, E. (2020). La producción de una franja fronteriza controlada. El ordenamiento territorial en el sureste mexicano. En Fenner, J., Lerma Rodríguez, E., Piedrasanta Herrera, R. \& Torras Conangla, R. (Coords.), Vidas transfronterizadas: dinámicas y actores en el límite Guatemala/México, siglo XIX-XXI (pp. 169-199). Cimsur-UNAM.

Lerma, E. \& Monterrosa, L. G. (2020). Notas introductorias a la obra de Ricardo Falla. Resultados de una jornada de estudio en Chiapas. Revista Pueblos y Fronteras Digital, 15, e499. http://www.pueblosyfronteras.unam.mx/index.php/index. $\mathrm{php} / \mathrm{pyf} /$ article/view/499

Leyva, X. \& Ascencio, G. (2002). Lacandonia al filo de agua. Universidad Nacional Autónoma de México/Centro de Investigaciones y Estudios Superiores en Antropología Social/Fondo de Cultura Económica.

Leyva, X. \& Burguete, A. (2004). Estudios monográficos. Nuevos municipios en Chiapas. Centro de Investigaciones y Estudios Superiores en Antropología Social/Gobierno de Chiapas.

Leyva, X. \& Burguete, A. (Eds.). (2007). Remunicipalization in Chiapas. Politics and the political in times of counter-insurgency. Centro de Investigaciones y Estudios Superiores en Antropología Social/International Work Group for Indigenous Affairs. https:// www.iwgia.org/en/documents-and-publications/documents/publications-pdfs/ english-publications/241-mexico-web/file.html

Limón, F. (2009). Historia chuj a contrapelo. Huellas de un pueblo con memoria. El Colegio de la Frontera Sur.

Martínez Velasco, G. (1994). Plantaciones, trabajo guatemalteco y política migratoria en la frontera sur de México. Gobierno del Estado de Chiapas.

Medina, A. (1973). Notas etnográficas sobre los mames de Chiapas, Méx. Anales de Antropología, 10, 141-220. http://www.revistas.unam.mx/index.php/antropologia/article/view/23286 
Moctezuma Navarro, D. (Coord.). (1994). Chiapas. Los problemas de fondo. Centro Regional de Investigaciones Multidisciplinarias-UnAM.

Ordoñez, C. E. (1994). Modernización y desarrollo regional en Chiapas. Un caso: la zona libre de Tapachula. Centro de Investigaciones Humanísticas de Mesoamérica y el Estado de Chiapas/unam.

Ordoñez, C. E. (2001). Tendencias de la integración económica fronteriza en Chiapas y Guatemala. En O. Gall (Coord.), Chiapas: sociedad, economía, interculturalidady política (pp. 269-295). Centro de Investigaciones Interdisciplinarias en Ciencias y Humanidades-UnAm.

Piedrasanta Herrera, R. (2009). Los chuj. Unidad y rupturas en su espacio. ARMAR Editores.

Ramírez López, D. K. (2018). Mujeres migrantes en la frontera sur de México: aproximaciones desde la insterseccionalidad. Centro Regional de Investigaciones Multidisciplinarias-UNAM.

Ramos Rojas, D. N. (2020). La triple frontera: propuesta conceptual para explicar las dinámicas de la región fronteriza entre México y Guatemala. Cuadernos Inter.c.a.mbio sobre Centroamérica y el Caribe, 17(2), e43760. https://revistas.ucr. ac.cr/index.php/intercambio/article/view/43760/44052

Rivera, C. (Coord.). (2014). Trabajo y vida cotidiana de centroamericanos en la frontera suroccidental de México. ciesas/Publicaciones de La Casa Chata.

Rodríguez, L. (2017). Maravilla Tenejapa. Monografía municipal. Edición del autor.

Rojas Pérez, H. S. \& Fletes Ocón, H. B. (2017). Configuración regional del Estado: orden mercantil y comunidad interpretativa en la frontera México-Guatemala. Estudios Fronterizos, 18(35), 1-21. https://doi.org/10.21670/ref.2017.35.a01

Rojas Wiesner, M. \& Ángeles Cruz, H. M. (2012). La situación de las mujeres migrantes en la frontera de México con Guatemala. En E. Tuñón Pablos \& M. L. Rojas Wiesner, (Coords.), Género y migración (pp. 37-66). El Colegio de la Frontera Sur/El Colegio de la Frontera Norte/El Colegio de Michoacán/Centro de Investigaciones y Estudios Superiores en Antropología Social.

Roldán, Ú. (2017). La frontera estratégica y extendida, México-Centroamérica. Sus impactos: "seguridad nacional versus derechos humanos de la población migrante”. En A. Ares \& J. Eguren (Eds.), Los movimientos migratorios en las fronteras iberoamericanas (pp. 163-190). Observatorio Iberoamericano Movilidad Humana, Migraciones y Desarrollo. https://www.academia.edu/33065574/_2017_ Los_Movimientos_Migratorios_en_las_Fronteras_Iberoamericanas

Ruíz Lagier, V. (2013). Ser mexicano en Chiapas. Identidad y ciudadanización entre los refugiados guatemaltecos en La Trinitaria, Chiapas. INAH.

Ruíz Lagier, V. (2018). Los refugiados guatemaltecos y la frontera-frente de discriminación, explotación y desigualdad. Revista Alteridades, 28(56), 47-57. https://doi. org/10.24275/uam/izt/dcsh/alteridades/2018v28n56/Ruiz

Sepúlveda, C. (1958). Historia y problema de los límites de México iI: la frontera sur. Historia Mexicana, 8(2), 145-174. https://historiamexicana.colmex.mx/index. $\mathrm{php} / \mathrm{RHM} /$ article/view/757

Tamayo, L. M. O. (2015). La Comisión Mexicana de Límites y la definición de la frontera sur del país. Revista de Geografia Norte Grande, (60), 115-134. https://www. redalyc.org/pdf/300/30041118007.pdf 
Toledo-Pineda, M. Á. \& Coraza de los Santos, E. (2019). Los mam de México y Guatemala: un pueblo binacional entre la autonomía y la heteronomía. Revista Pueblos y Fronteras Digital, 14. https://doi.org/10.22201/cimsur.18704115e.2019.v14.369

Valdez, M. (2006). Desencuentro y encuentro de fronteras: el Petén guatemalteco y el sureste mexicano 1895-1949. Universidad intercultural de Chiapas-Universidad de Ciencias y Artes de Chiapas.

Villafuerte Solís, D. (2004). La frontera sur de México. Del TLC México-Centroamérica al Plan Puebla-Panamá. Plaza y Valdés/Instituto de Investigaciones Económicas-UNAM.

Villafuerte, D. \& García, M. C. (2005). Las fronteras de la frontera sur. En A. Basal Rodríguez (Coord.), Fronteras des-bordadas. Ensayos sobre la frontera sur de México (pp. 123-151). Editorial Juan Pablos/Universidad de Ciencias y Artes de Chiapas.

Viqueira, J. P. \& Ruz, M. H. (2004). Chiapas. Los rumbos de otra historia. Centro de Investigaciones y Estudios Superiores en Antropología Social/unAm.

Enriqueta Lerma

Mexicana. Doctora en antropología por la Universidad Nacional Autónoma de México, miembro del Sistema Nacional de Investigadores, nivel 1. Investigadora del Centro de Investigaciones Multidisciplinarias sobre Chiapas y la Frontera Sur, de la Universidad Nacional Autónoma de México. Líneas de investigación: conceptualizaciones culturales sobre el territorio; desarrollo comunitario y autonomía; religión en espacialidades fronterizas. Publicación reciente: Fenner, J., Lerma Rodríguez, E., Piedrasanta Herrera, R. \& Torras Conangla, R. (Coords.). (2020). Vidas transfronterizadas: dinámicas $y$ actores en el límite Guatemala/México, siglos XIX-XXI. Cimsur-UnAM.

\section{Perla Mónica Castro Cruz}

Mexicana. Estudiante de antropología en la Facultad de Ciencias Políticas y Sociales de la Universidad Nacional Autónoma de México. Líneas de investigación: antropología de las fronteras y género. Participa como colaboradora en la revista digital independiente Voces de Quimeras y es miembro del proyecto PAPIIT IN300620 “La producción de la frontera Chiapas-Guatemala: una aproximación al ordenamiento territorial y a su resignificación". 